\section{It's about (molecular) time}

\begin{tabular}{ll} 
& $\begin{array}{l}\text { Molecular Biology of Circadian } \\
\text { Rhythms } \\
\text { Edited by Amita Sehgal }\end{array}$ \\
\hline $\begin{array}{l}\text { MOLECULAR } \\
\text { BIOLOGY } \\
\text { CIRADIA } \\
\text { RHYTHMS }\end{array}$ & John Wiley \& Sons $\bullet 2004$ \\
& $\$ 89.95 / £ 52.95$ \\
\hline & \\
& Ravi Allada
\end{tabular}

The sunrise is one of the most predictable events in our environment. It should come as no surprise then that myriad organisms have evolved internal biological timers, or circadian clocks, to anticipate this and other daily transitions. As a result, much of our behaviour and physiology is temporally synchronized with these daily environmental changes.

The past decade has witnessed stunning advances in our molecular understanding of these circadian clocks. The field has also experienced growth, both from within and due to immigration into it. A comprehensive description no longer fits conveniently into the typical review article, and thus, the time is right for a book that compiles our accumulated knowledge on molecular clocks. Molecular Biology of Circadian Rhythms, containing chapters authored by various researchers in the field, aims to provide such a comprehensive guide.

For new entrants to circadian biology, part I of the book is an excellent introduction to our unique jargon that describes essential concepts, but can also, unfortunately, exclude the uninitiated. The first chapter defines classical concepts, such as phase response curve, state variable and Zeitgeber, that describe the clock and its synchronization (or entrainment) to environmental cycles. The second chapter discusses the most commonly used techniques in molecular circadian biology. Although this book cannot replace a basic text on molecular biology, it attempts to make molecular concepts comprehensible to a non-molecular audience. In subsequent chapters this approach is not uniform and therefore some background in molecular biology is recommended to fully exploit this text. Nonetheless, these first chapters are highly accessible to a broad audience.

One of the most extraordinary aspects of circadian biology research is its biodiversity; that is, the range of organisms that serve as experimental models. A biological rhythms meeting is one of the few places that one finds scientists who work on photosynthetic bacteria rubbing shoulders with those studying human sleep. Parts II and III of the book describe the current state of circadian biology research in multiple model systems including fruit flies, non-mammalian vertebrates, mammals, photosynthetic bacteria, fungi and plants.
Applying genetics to understand circadian behaviour represents a triumph not just for circadian biology but also in the broader endeavour to link specific genes to behaviour. This story begins with Seymour Benzer's pioneering screens of populations of fruit flies randomly mutagenized for altered circadian rhythms, and the subsequent identification of the period (per) mutants. The cloning of the per gene, along with the Neurospora crassa frequency gene, ultimately led to the identification of the central organizing principle of clocks: transcriptional feedback loops. Later, a behavioural genetic approach inspired by Benzer identified the first circadian rhythm gene in mammals, the Clock gene. The subsequent discovery of a plethora of shared clock components firmly solidified the notion that insect and mammalian clocks are evolutionarily coupled. Thirty years after the isolation of the fruit fly per mutants, the identification of a mutation in a human homologue of per, in individuals affected by a circadian sleep syndrome, indicated that studies of the lowly fruit fly might actually tell us something about how we sleep. The coverage of all these model systems in the book is inclusive and it offers an in-depth explanation of the classic experiments that led to major discoveries. The relative merits of the different model systems are also well discussed. Moreover, this section of the book serves as a road map to discovering the genetic basis of any complex behaviour and should therefore also interest behavioural geneticists.

One of the most unexpected and far-reaching findings that emerged from the discovery of the clock genes was that clocks are everywhere - in most organs and tissues, and even in some commonly used cell lines. This has led to the idea that circadian rhythms are not merely driven by master clocks in the brain, but are the result of dedicated clock programmes within cells. Understanding how these multi-oscillatory systems operate is the subject of part IV. Scientists working in almost any physiological system should find the description of these so-called 'peripheral clocks' compelling.

Understanding how core molecular oscillators control cellular function to influence behaviour, physiology and, most importantly, disease is a major issue. Studies revealing unexpected connections of circadian genes to drug addiction and cancer will be of keen interest to those working on disease models. Although the section on human circadian rhythms does not discuss their molecular basis, it is especially well written and it should be relevant to applying our new-found understanding of clocks to human biology and disease. However, there was a lack of discussion on connections between circadian rhythms and common human diseases, including affective, pulmonary and cardiovascular disorders. Elucidating the molecular pathways connecting clocks to these diseases will undoubtedly be an area of active investigation.

Molecular Biology of Circadian Rhythms is an excellent and comprehensive introduction to circadian biology, especially appropriate for those starting work in this area, advanced undergraduate and graduate students, and even aficionados who want a general brush up on the whole field. 